\title{
Fundamentos de la tecnología biofloc (BFT). Una alternativa para la piscicultura en Colombia. Una revisión
}

\author{
Fundamentals of bioflocs technology (BFT). \\ An alternative for fish farming in Colombia. A review.
}

\author{
Fundamentos da bioflocos tecnologia (BFT). \\ Uma alternativa para a piscicultura na Colômbia. Uma revisão.
}

\author{
Luis F. Collazos-Lasso ${ }^{1}$, José A. Arias-Castellanos ${ }^{2 *}$
}

1 Ingeniero en Producción Acuícola, MSc, Estudiante de Doctorado en Ciencias Agrarias;

2 Biólogo, MSc, PhD.

* Instituto de Acuicultura de los Llanos - IALL, Facultad de Ciencias Agropecuarias y Recursos Naturales, Universidad de los Llanos, Villavicencio, Meta - Colombia.

** Fundación Orinoquia, Puerto Carreño, Vichada - Colombia

Email: Ifclasso@yahoo.com

Recibido: diciembre 12 de $2014 \quad$ Aceptado: junio 04 de 2015

\begin{abstract}
Resumen
Los sistemas convencionales de producción piscícola en Colombia empiezan a descender principalmente por la necesidad de grandes cantidades de agua cada vez más escasa, aumento de la contaminación de los afluentes de descargue, aumento del costo de los alimentos con gran desperdicio de los mismos y otros factores ambientales adversos como sequías en grandes áreas del territorio e irregulares volúmenes de producción por unidad de área o volumen. Por lo anterior la búsqueda de nuevas posibilidades de producción piscícola que sean amigables con el ambiente, incluyentes socialmente y rentables son cada vez más apremiantes. Una de las alternativas que empiezan a cautivar el interés de los piscicultores es el sistema de producción súper-intensiva con tecnología biofloc (BFT), la cual se sustenta en aprovechar la acumulación de residuos de los alimentos, materia orgánica y compuestos inorgánicos tóxicos a través de microorganismos presentes en los medios acuáticos, dando condiciones de dominancia a comunidades autótrofas y heterótrofas, resolviendo sustancialmente los problemas de saturación de nutrientes a partir de su reciclaje, en este sentido el objetivo de la presente revisión es presentar los fundamentos básicos de la BFT, como una alternativa de producción piscícola.
\end{abstract}

Palabras clave: microorganismos, tecnología biofloc, piscicultura, nutrientes.

\begin{abstract}
Conventional systems for fish production in Colombia begin to decline mainly due to the need for large quantities of increasingly scarce water, increasing pollution of the tributaries of discharge, increased cost of food with great waste of these and other factors adverse environmental and droughts in large areas of territory and different volumes of production per unit of area or volume. Therefore the searches for new potential for fish production is environmentally friendly, socially inclusive and profitable are becoming more pressing. One of the alternatives that are beginning to captivate the interest of farmers is the system of super intensive production bioflocs technology (BFT), which is based on the accumulation of waste
\end{abstract}


seize food, organic and inorganic toxic compounds through of microorganisms in aquatic environments, giving dominance conditions and heterotrophic to autotrophi production.

Keywords: microorganisms, bioflocs technology, fish production, nutrients.

\section{Resumo}

Os sistemas convencionais de produção de peixes na Colômbia começam a diminuir, devido, principalmente, à necessidade de grandes quantidades de água cada vez mais escassa, aumentando a poluição dos afluentes da descarga, aumento do custo de alimentos com grande desperdício de estes e outros fatores adverso ambiental e secas em grandes áreas de território e os volumes irregulares de produção por unidade de área ou volume. Por isso, a busca por um novo potencial para a produção de peixes que são respeitadores do ambiente, socialmente inclusiva e rentável é cada vez mais premente.

Uma das alternativas que estão começando a cativar o interesse dos agricultores é o sistema de superintesivo tecnologia de produção bioflocos (BFT), que se baseia na acumulação de resíduos aproveitar alimentos, orgânicos e inorgânicos por meio de compostos tóxicos de microrganismos em ambientes aquáticos, dando condições de dominância e heterotróficos às comunidades autotrophic resolver substancialmente os problemas de saturação de nutrientes provenientes da reciclagem, nesse sentido, o objetivo desta revisão é apresentar os conceitos básicos da BFT, como uma alternativa para a produção de peixe.

Palavras-chave: microorganismos, bioflocos tecnología, produção de peixes, nutrientes.

\section{Introducción}

En la actualidad la comunidad mundial se enfrenta a retos relacionados con atender las necesidades apremiantes de alimentación y nutrición de una población creciente con recursos naturales finitos (FAO, 2012), por ello la Organización Mundial de la Salud (WHO 2003) dentro de sus estrategias recomendó el aumento del consumo de pescado para mejorar las expectativas de vida y salud de las personas, en consecuencia en los últimos años el interés en los productos de la acuicultura ha aumentado, lo cual se ve reflejado en las cifras de producción acuícola mundial reportadas para peces comestibles, donde se muestra un incremento en la tasa media anual del $8.6 \%$ desde la década del ochenta llegando a 66,6 millones de TM en el 2012 (FAO, 2014), en América Latina tal incremento llega al 10\% en 2010 (1,9 millones de TM).

En un contexto nacional la producción piscícola en Colombia ha tenido un crecimiento promedio anual desde 1990 del 12\%, llegando en el 2011 a 74.270 $\mathrm{TM}$, de las cuales $99,9 \%$ provienen de la piscicultura continental, siendo las tilapias las más producidas con un $65 \%$, seguido por la cachama blanca (Piaractus brachypomus) con un $21 \%$, de estos datos el $66 \%$ del volumen total de producción corresponden a cultivos semintensivos en estanques en tierra y $34 \%$ cultivos intensivos en jaulas (AUNAP, 2013).

El aumento de la producción de peces en Colombia, como en el resto del mundo, también ha generado crecientes problemas, el principal de ellos la eutrofización por descargas de nutrientes, componentes orgánicos e inorgánicos (amonio, fósforo, materia orgánica, carbo- no orgánico disuelto y sólidos suspendidos), los que son responsables de la polución, nitrificación y enterramiento de comunidades bentónicas en los ecosistemas receptores (Martínez et al., 2010). El segundo conflicto que genera la piscicultura en Colombia es el uso de grandes volúmenes de agua con producciones irregulares y relativamente bajas por unidad de volumen, aspecto ambientalmente adverso que unido a la pérdida creciente de oferta de agua nacional vuelven los sistemas piscícolas extremadamente frágiles.

Por lo anterior, se han implementado en el mundo y en el país nuevas formas de producción más amigables con el ambiente (menos contaminantes y más ahorradoras de agua entre otras propiedades), las recientes tecnologías irrumpen prometedoras porque además son más eficientes productivamente aunque de ellas se conozca muy poco y no se sepa de muchos aspectos técnicos y de otros insumos importantes para esos sistemas (Atencio et al., 2013).

Una de las más atractivas tecnologías es la de los biofloc, la cual se basa en aprovechar los residuos de los alimentos, materia orgánica y compuestos inorgánicos tóxicos (los cuales conlleva al deterioro de la calidad del agua y al poco aprovechamiento del alimento natural), a través de microorganismos presentes en los medios acuáticos, dando condiciones de dominancia a comunidades bacterianas quimio / foto autótrofos y heterótrofas, resolviendo así sustancialmente los problemas de saturación de nutrientes a partir de su reciclaje (Avnimelech, 2009). 
Esta revisión pretende colocar en un solo escrito los avances hasta el momento relacionados con los diferentes aspectos de orden técnico que gobiernan el mundo de los biofloc y de sus uso para la producción acuícola, como una contribución al estudio, comprensión y desarrollo de los mismos.

\section{Generalidades de la tecnología biofloc}

El trabajo clave que dio inicio al estudio de la tecnología fue el de Azam et al., 1983, en el que se hace un acercamiento al papel y la dinámica que cumplen los microorganismos en un sistema acuático natural, en este sentido estos autores plantean el aprovechamiento por el "microcosmos acuático", del carbono dispuesto en el agua en condiciones ricas en nitrógeno, comprobando que las bacterias fijan carbono como fuente de energía y aprovechan el nitrógeno para la síntesis de proteínas; bajo este supuesto el denominado 'microbial loop', término acuñado en el artículo en cuestión, incluye el papel desempeñado por las bacterias en relación con el carbono y los ciclos de nutrientes (red trófica microbiana), la que se caracteriza por reciclar nutrientes. La dinámica general es el resultado de varias relaciones ecológica (comensalismo, competencia, depredación entre otras), siendo entonces una micro-red trófica paralela a la cadena trófica convencional; el principio productivo nace del consumo directo del carbono contenido en la materia orgánica disuelta (MOD), por las bacterias heterotróficas y el pico-plancton $(10-20 \mu)$, biocarbono que se produce en los ecosistemas tras las primeras etapas de degradación de la materia orgánica (excrementos, restos de plantas, organismos muertos etc.), son por tanto organismos consumidores, que son alimento a su vez, de otros microorganismos (flagelados y ciliados por ejemplo), construyéndose así en entramado trófico.

Las explicaciones dadas a las interacciones de ese pequeño cosmos en ambientes acuáticos naturales dio inicio a investigaciones que buscaban contrarrestar la acumulación de compuestos nitrogenados especialmente amonio y nitritos en los sistemas de producción de especies acuáticas, así nació el concepto de biofloc, como el de una comunidad constituida de microorganismos asociados entre sí en un sustrato suspendido o flotante que responde a una dinámica de malla trófica que se inicia en heterótrofos capaces de fijar carbono desde las sustancias y partículas orgánicas en el agua y cuya densidad se sitúa entre 10 y 1.000 millones de células microbianas / $\mathrm{cm}^{3}$ (Burford et al., 2004). La comunidad de biofloc es de forma irregular, deformable, porosa, de tamaño indefinido (desde pocas micras hasta varios centímetros de diámetro), y más denso que el agua por lo que tienden a sedimentarse lentamente (Martínez et al., 2010). Funcionalmente es un complejo donde ocurren al mismo tiempo actividades autotróficas y heterotróficas utilizando aportes exógenos (Ebeling et al., 2006). Cada biofloc es también un micronicho con necesidades fisiológicas particulares según este agregado y en el que cohabitan procesos complementarios aeróbicos y anaeróbicos siendo las interacciones que se producen piezas claves para el mantenimiento de la calidad de las aguas (Ray et al., 2010; Okabe y Watanabe, 2000).

Teniendo en cuenta lo anterior el uso y cultivo de los biofloc microbianos a partir de una alta relación de $C: N$ en el agua, ha sido empleado para acuicultura como un sistema alternativo super-intensivo de producción, siendo que el nitrógeno proviene del alimento no consumido y de la excreción propia de la especie de cultivo y el carbono de la adición de una fuente externa de carbohidratos, con poco o nulo recambio de agua y una alta oxigenación (Emerenciano et al., 2013; Monroy-Dosta et al., 2013; Avnimelech, 2012a; Craig et al., 2012; Emerenciano et al., 2012; Kubitza, 2011).

El desarrollo de los conceptos y aplicaciones referidas han dado origen al abreviado BFT(del inglés Bio-Floc Tecnology), que además se fundamenta en mantener las condiciones de calidad del agua en relación con la fijación y control del nitrógeno inorgánico toxico $\left(\mathrm{NH}_{4}, \mathrm{NH}_{3}, \mathrm{NO}_{2}\right.$ y NO3), y en generar "in situ", proteína microbiana aprovechable como alimento por la especie cultivada (Ekasari et al., 2014; Emerenciano, 2013; Monroy-Dosta et al., 2013;Craig et al., 2012; Kubitza, 2011; Avnimelech, 2009; De Schryver et al., 2008; Azim et al., 2008; Hari et al., 2004).

La BFT es una forma de producción en acuicultura super-intensiva, que se desarrolla dinámicamente en la actualidad pues resulta que es capaz de enfrentar retos propios de la actividad, como el aumento de la biomasa por volumen de agua y la utilización cada vez más reducida de agua, el desafío en concreto es producir más en menos volumen de agua y al menor costo ambiental posible, es decir en el marco de los paradigmas de sostenibilidad (Avnimelech, 2009). Aunque falta mucho por conocer, el hecho que la BFT trate conceptualmente los residuos como una oportunidad de producción in situ, lo hace una alternativa posible y amigable con los ecosistemas porque al tiempo que economiza agua y recicla nutrientes, descarga pocos contaminante (Wasielsky, 2006). 


\section{Sobre los compuestos nitrogenados en acuicultura}

Como se sabe todos los sistemas de producción piscícola generan desperdicios (constituidos por material sólido, alimento no consumido, heces y materiales solubles como fósforo y nitrógeno), y productos de excreción (de los peces en cultivo), en especial esto es cierto cuando se usa grandes raciones para la alimentación, agudizándose con el aumento de la biomasa que demanda más alimento (Sagratzki et al., 2004; Gelineau et al., 1998).

El $\mathrm{N}$ puede estar presente en los ambientes acuáticos en formas de nitrato $\left(\mathrm{NO}_{3}{ }^{-}\right)$, nitrito $\left(\mathrm{NO}_{2}{ }^{-}\right)$, amonio ionizado $\left(\mathrm{NH}_{4}^{+}\right)$, amonio no ionizado $\left(\mathrm{NH}_{3}\right)$, óxido nitroso $\left(\mathrm{N}_{2} \mathrm{O}\right)$, óxido nítrico $(\mathrm{NO})$, nitrógeno molecular $\left(\mathrm{N}_{2}\right)$, nitrógeno orgánico disuelto (péptidos, purinas, aminas, aminoácidos) y como nitrógeno orgánico particulado (Hernández y Vargas, 2003), de todas estas formas de nitrógeno, los nitratos y el amonio son los más importantes para los ecosistemas acuáticos, por cuanto constituyen la fuente principal de $\mathrm{N}$ biodisponible para la generación de cadenas tróficas, siendo que el amonio $\left(\mathrm{NH}_{3}\right)$ y el nitrito $\left(\mathrm{NO}_{2}\right)$ son tóxicos para los peces y se convierten en un factor limitante para el crecimiento y sobrevivencia de estos en cultivo, así removerlo o transformarlo en nitrógeno no toxico es esencial cuando se pretende aumentar la biomasa del sistema y disminuir los riesgos (Avnimelech, 2009; Ebeling y Timmons, 2006; Hargreaves, 1998).

Los niveles de proteína del concentrado suministrado normalmente en acuicultura oscilan entre el 20 y $45 \%$, del cual aproximadamente el $16 \%$ es nitrógeno (Craig y Helfrich, 2002), y de este cerca del $75 \%$ es aportado al medio de cultivo por excreción y alimento no consumido (Craig et al, 2012; Avnimelech, 2009; De Schryver, 2008; Piedrahita, 2003; Hargreaves, 1998). El metabolismo del alimento ingerido termina con la formación de amonio ionizado y no ionizado que es excretado principalmente por las branquias al agua, la suma de estas formas de amonio $\mathrm{NH}_{4}^{+}+\mathrm{NH}_{3}$ se le denomina Nitrógeno Amoniacal Total (NAT), en cultivos de peces la forma no ionizada $\mathrm{NH}_{3}$ es altamente toxica y la concentración letal varía entre especies en un rango de 1 - $2 \mathrm{mg} / \mathrm{L}$, agudizándose cuando la concentración de oxígeno es baja (Avnimelech, 2009), el aumento del amonio no ionizado depende también del aumento del $\mathrm{pH}$, de la temperatura y de la salinidad (Ebeling et al., 2006; Timmons et al., 2002), en presencia de microorganismos fotoautótrofos las concentraciones de $\mathrm{NH}_{3}$ aumentan en horas de la tarde cuando el $\mathrm{pH}$ y la temperatura están en niveles altos y el $\mathrm{CO}_{2}$ es mínimo.

\section{Ruta de los compuestos nitrogenados en cultivos biofloc}

La BFT busca maximizar el potencial de los procesos microbianos dado a que la variedad de bacterias en un contenedor, son capaces de degradar las diferentes formas de nitrógeno incluidas las más nocivas para los peces (Avnimelech, 2009). Tres grupos de microbíota de remoción de los compuestos nitrogenados del agua son ampliamente conocidas, todas ellas en diferente grado pueden interactuaren sistemas biofloc, así: asimilación por algas, oxidación por bacterias quimioautótrofas y asimilación por bacterias heterotróficas (Ray y Lotz, 2014); a las cuales si se les suma otros organismos como zooplancton, hongos y nematodos, todos abundantes por la casi infinita capacidad reproductiva que poseen, los cuales en conjunto consiguen el control casi absoluto de los desechos del nitrógeno (Monroy-Dosta et al., 2013; Wilén et al., 2008; Jorand et al., 1995).

Tanto en ambientes acuáticos naturales como en cultivo, los organismos relacionados con el ciclo del nitrógeno recuperan los nutrientes volcados a las aguas, disminuyendo los compuestos nitrogenados tóxicos al degradar los restos de alimento no consumido, las excretas y heces (Moss, 2002), siendo los mayores productores acuáticos (hasta el $70 \%$ de la productividad total de cualquier cuerpo de agua) (Crab et al., 2010), manteniendo las calidades de las mismas (Tzachi et al., 2012; Ebeling et al., 2006), sirviendo de alimento a la gigantesca red trófica que nace de ellos y que termina nutriendo los peces en cultivo (Abreu et al., 2007) y controlando los patógenos (De Schryver et al., 2008, Crab et al., 2007). El conjunto de toda las formas vivas asociadas y relacionadas con un sin número de partículas orgánicas e inorgánicas con las que forman películas en las paredes de los contenedores o aglomerados amorfos suspendidas en la columna de agua (biofloc), los cuales se mantienen unidos por una matriz de mucosidad que es secretada por las propias bacterias y los microorganismos filamentosos que los componen y por atracción electrostática (Avnimelech et al., 2008, De Schryver et al., 2008).

La BFT entonces en la práctica consiste en el manejo de las comunidades microbianas, ello es lo que determina el éxito del sistema basado en la transformación de los compuestos nitrogenados en el agua, asunto que según Ebeling et al., (2006), se consigue de diferentes maneras, como se presenta a continuación:

Las bacterias heterotróficas presentan la siguiente reacción metabólica que incluyen la descomposición del amonio para transformarlo en biomasa bacteriana: 


$$
\begin{gathered}
\mathrm{NH}_{4}+\mathrm{C}_{6} \mathrm{H}_{12} \mathrm{O}_{6}+\mathrm{HCO}_{3}+\mathrm{O}_{2} \rightarrow \mathrm{C}_{5} \mathrm{H}_{7} \mathrm{O}_{2} \mathrm{~N}+\mathrm{H}_{2} \mathrm{O} \\
+\mathrm{CO}_{2}
\end{gathered}
$$

El balance estequimétrico de las reacciones anteriores indicará que para remover $1 \mathrm{~g}$ de amonio, el consumo de carbohidratos será igual a 15,2 g, alcalinidad $=3,6$ g y oxígeno $=4,7 \mathrm{~g}$, produciendo sólidos en suspensión volátiles $(\mathrm{SSV})=8 \mathrm{~g}$ y $\mathrm{CO}_{2}=9,7 \mathrm{~g}$. Dicho en otros términos por cada gramo de NAT producido en un tanque de cultivo, es necesario añadir aproximadamente $\approx 20$ gramos de hidratos de carbono (C: $\mathrm{N}$ de 20:1) (Avnimelech, 1999), una consecuencia directa de la adición de carbohidratos para lograr estas proporciones es el aumento de la demanda de oxígeno disuelto, atribuido a las reacciones químicas propias de las bacterias heterótrofas (Schveitzer et al., 2013).

Para las bacterias quimioautotróficas, las reacciones de metabolismo incluyen la descomposición del amonio $\left(\mathrm{NH}_{3}\right)$ en nitrito $\left(\mathrm{NO}_{2}\right)$ y después en nitrato $\left(\mathrm{NO}_{3}\right)$, para finalmente a través de reacciones anaeróbicas por proceso de des-nitrificación, en nitrógeno atmosférico $\left(N_{2}\right)$ :

\section{$\mathrm{NH}_{4}+\mathrm{O}_{2}+\mathrm{HCO}_{3} \rightarrow \mathrm{C}_{5} \mathrm{H}_{7} \mathrm{O}_{2} \mathrm{~N}+\mathrm{NO}_{3}+\mathrm{H}_{2} \mathrm{O}+\mathrm{CO}_{2}$}

El balance de las anteriores reacciones indicará que para transformar 1 gramo de amonio, el consumo de alcalinidad $=7,0 \mathrm{~g}$; oxígeno $=4,2 \mathrm{~g}$ y se produce SSV $=0,2 \mathrm{~g} ; \mathrm{CO}_{2}=5,9 \mathrm{~g}$ y $\mathrm{NO}_{3}=0,98 \mathrm{~g}$.

El análisis de las dos reacciones anteriores muestra que las bacterias quimioautotróficas nitrificantes presentes en el biofloc requieren menos carbohidratos para la transformación y remoción de nitrógeno, con la consecuente disminución en la demanda de oxígeno (Avnimelech, 2006; Ebeling et al., 2006), en este sentido la relación C:N sería de 15 - 10: 1 (Avnimelech, 2012 ${ }^{\mathrm{b}}$ ), siendo que las dos vías (bacterias autótrofas y heterótrofas)para la eliminación de nitrógeno son diferentes en términos de la utilización del sustrato, la biomasa bacteriana que generan y los subproductos que producen, sin embargo debido a la menor velocidad de reproducción de las bacterias nitrificantes, se necesita la presencia de bacterias heterótrofas especialmente en los primeros días de cultivo para asegurar el secuestro y la reducción de amoníaco producido. Las bacterias quimioautotróficas necesitan alrededor de 30 días para su establecimiento en el tanque de cultivo (Timmons y Ebeling, 2010).

\section{De las relaciones $\mathrm{C}: \mathrm{N}$ en $\mathrm{BFT}$}

El ambiente natural propicio que ayude y posibilite la presencia de células microbianas debe tener una re- lación Carbono: Nitrógeno aproximada de 5:1 (Goldman et al., 1987 en: Hargreaves, 2006), las bacterias se alimentan con sustrato orgánico que contiene principalmente carbono y nulo o poco nitrógeno, este último lo toman del agua con el fin de producir la proteína necesaria para el crecimiento y la multiplicación celular. En sistemas de producción acuícola el control de la acumulación de nitrógeno inorgánico en el contenedor se basa en el metabolismo del carbono y la inmovilización de nitrógeno por células microbianas (Avnimelech, 2012ㄹ ; Avnimelech, 1999), las bacterias y otros microorganismos usan carbohidratos (azúcar, almidón y celulosa) como alimento, para la generación de energía y crecimiento.

En consecuencia, para que las comunidades bacterianas autótrofas y/o heterótrofas prosperen debe haber disponibilidad del carbono inorgánico y orgánico, sin embargo para el caso de las bacterias heterótrofas, Ebeling et al., (2006), sugieren que no todo el carbono orgánico en la alimentación es fácilmente disponible para las bacterias, por ejemplo, sólo109g / kg de carbono orgánico lábil está contenido en un alimento con 35\% de proteína, en comparación con un análisis aproximado de 350-400 g de carbono / kg alimento. La capacidad de controlar el tipo de carbono y la relación C: $\mathrm{N}$, por la formulación del alimento, eliminación o adición de sólidos orgánicos de carbono permite a los técnicos y productores acuícolas definir qué tipo de vía se vuelve dominante en sus sistemas de producción, en este sentido las relaciones $\mathrm{C}$ : $\mathrm{N}$ han variado entre autores reportando tasas $>10: 1$ (Azim et al., 2008), 12 -13:1 (Schneider et al., 2006); 15:1 (Monroy, 2013), 20:1 (Poli et al., 2015; Ekasari, 2014; Emerenciano, 2012; Emerenciano et al., 2012; Craig et al., 2012; Avnimelech, 2009; De Shryver 2008; Avnimelech, 1999).

Por lo anterior, los aportes de restos nitrogenados de los peces (excreción + alimento no consumido), al disolverse desequilibran la relación del nitrógeno con el carbono, para controlar tal desequilibrio la BFT propone agregar algún material rico en carbono soluble, uno o una mezcla de carbohidratos (Samocha et al., 2007).

La cantidad de carbohidrato suplementario requerido para mantener reducido el amonio depende de la cantidad de alimento suministrado(relacionado con la biomasa), las estimaciones deben entonces tener en cuenta el porcentaje de proteína del alimento, el porcentaje de nitrógeno presente en la proteína (aproximadamente del $\approx 16 \%$ ), y el porcentaje de excreción de dicho nitrógeno $(75 \%$ por excreción + alimento no consumido) (De Schryver, 2008), descontado previa- 
mente el porcentaje de humedad propio de cada alimento, aspecto que no siempre se considera.

Avnimelech (2009), De Schryver (2008) y Kubitza, (2011), plantean que el porcentaje de carbón que tienen las diferentes fuentes se aproxima al $\approx 50 \%$, siendo la más utilizada la melaza (Monroy et al., 2013; Emerenciano, 2012; Kuhn y Lawrence, 2012; Avnimelech, 2011; Schneider et al., 2006; Bufort et al., 2003), sin embargo el trabajo de Ray y Lotz (2014), estima dicho porcentaje de carbono de diferentes fuentes las cuales presentan variaciones (valores diferentes a $50 \%$ ), y como era de esperarse la eficiencia en cuanto a remoción del NAT fue diferente, afectando la alcalinidad la cual descendió, al parecer una respuesta común en comunidades heterotróficas dado que estas apelan para asimilar el nitrógeno al consumo de bicarbonatos como fuente de carbono, por consiguiente se debe analizar el porcentaje de $\mathrm{C}$ de la fuente de carbohidratos para calcular las proporciones $\mathrm{C}: \mathrm{N}$ y hacer las correcciones pertinentes en el sistema.

Otra consideración que se debe tener en cuenta para hacer los cálculos de adición de carbohidratos es que los niveles de proteína del concentrado suministrado tienen estimada una relación $\mathrm{C} / \mathrm{N}$, en este sentido un concentrado con un $16 \%$ de PB tiene una relación 20:1, siendo las condiciones iniciales de esta relación y sirven como un "factor de corrección" para las estimaciones (Avnimelech, 2009).

\section{El oxígeno en los BFT}

Se reconoce que para aumentar densidad en un sistema de producción acuícola se debe garantizar que los parámetros de calidad de agua estén en los rangos establecidos para la especie. Para Boyd (1998) y Vinatea (2004), las concentraciones de oxígeno disuelto en el agua es el principal limitante para el buen desempeño productivo de los peces, para lo cual existen diferentes formas de mejoramiento, que van desde la renovación de agua hasta equipos que incorporan oxígeno al agua.

En cultivos con BFT los sistemas de incorporación de oxígeno al agua son fundamentales, sin oxígeno suficiente y aún más en superávit no es posible construir biofloc. Los aireadores son los equipamientos más comunes utilizados para oxigenar el agua en contenedores con biofloc y ellos deben ser escogidos de tal manera que suplan tres necesidades principales: primero las necesidades de respiración de la especie cultivada (Hargreaves, 2013; Crab et al., 2012; Ray et al., 2010; Vinatea, 2004; Boyd y Clay, 2002; Browdy et al., 2001; Mcintosh, 2001; Avnimelech, 1999); segun- do la respiración y reacciones de nitrificación propias en la metabolización de compuestos nitrogenados tóxicos de los microorganismos contenidos en el sistema (Timmons et al., 2002), y tercero, debe mantener los biofloc en suspensión constante para evitar la decantación y acumulo de solidos que conduzcan a reacciones anaerobias que produzcan metabolitos tóxicos letales que puedan llegar a afectar el cultivo (Boyd y Clay, 2002).

Según Avnimelech (2009), es importante determinar el aireador para poder suplir el oxígeno que se necesita en el sistema de cultivo, garantizando de esta manera cumplir con las exigencias y requerimientos de oxígeno del sistema y la eficiencia en cuanto a gastos energéticos. Tres términos son usados para definir la capacidad de un aireador para tal fin:

a) Standard Oxygen Transfer Rate (SOTR) $\rightarrow \mathrm{Kg} \mathrm{O}_{2} /$ hr, definida, como la cantidad de oxígeno transferido por un aireador al agua a una temperatura de $20{ }^{\circ} \mathrm{C}$, iniciando en una concentración de $\mathrm{O}_{2}=0$, en un tiempo determinado.

b) Standard Aeration Efficiency (SAE) $\rightarrow \mathrm{Kg} \mathrm{O} 2 / \mathrm{Kw} /$ hr, definido como el SOTR dividido por el poder aplicado por el aireador, iniciando de una concentración de $\mathrm{O}_{2}=0$.

c) Actual Oxygen Transffered (AOT), definido como la cantidad de $\mathrm{O}_{2}$ en el estanque, teniendo en cuenta la concentración de $\mathrm{O}_{2}$ inicial.

En estudios realizados por Pasco (2015), se probaron 4 modelos de aireadores (splash, de paletas, turbina y blower), para determinar cuál era más eficiente en cultivos BFT de tilapia, concluyendo que el modelo de spalsh es mejor en cuanto a rendimiento y satisface los requisitos funcionales para su uso en este tipo de cultivos.

\section{Parámetros y condiciones de calidad del agua para cultivos con BFT}

La medición de los parámetros de calidad de agua en cualquier sistema de cultivo acuícola es necesaria para garantizar la aplicación de buenas prácticas de manejo del cultivo que garantice el éxito del mismo, tal cuestión es además vital en el caso de cultivos con BFT, puesto que conocidos los registros el análisis de los mismos permite siempre aplicar correctivos para procurar mantener los parámetros dentro de los rangos de confort de cada especie de pez cultivada (Collazos y Arias, 2007). 
Los parámetros básicos de medición en cultivos biofloc son: oxígeno, temperatura, $\mathrm{pH}$, alcalinidad, amonio y sólidos sedimentables.

Es conveniente el mantenimiento del oxígeno disuelto cercano a $6 \mathrm{mg} / \mathrm{L}$ con saturaciones mayores del $60 \%$, siendo que existe una relación inversa entre el consumo de oxígeno y el peso corporal, así como un efecto positivo de la temperatura del agua sobre la tasa metabólica para tilapia (Valbuena y Cruz, 2006), cachama blanca (Sastre et al., 2004) y yamú (Valbuena et al., 2006).

El pH y la alcalinidad en sistemas de producción con BFT normalmente permanecen estables en el agua con rangos y valores de $7-9$ y $>50 \mathrm{mg}$ de $\mathrm{CaCO} 3 / \mathrm{L}$ respectivamente, existiendo una relación directa entre los procesos de nitrificación y la alcalinidad e inversos en relación con el pH, en este sentido cuando el pH es alto promueve toxicidad por amonio no ionizado (Avnimelech, 2009), sin embargo una alcalinidad entre $40-100 \mathrm{mg} / \mathrm{L}$ de $\mathrm{CaCO}_{3}$ genera un efecto buffer que disminuye la oscilación del pH. También la temperatura del agua afecta al $\mathrm{pH}$ teniendo correlaciones positivas, es decir que mayor temperatura mayor $\mathrm{pH}$ y mayor amonio tóxico (Emerson, 1975).

El perfil de sólidos en un contenedor con biofloc es sin duda el indicador de calidad más propio del sistema. Diferentes tipos de sólidos existen en un tanque con biofloc, los más comunes y fáciles de determinar y con los cuales se puede administrar el cultivo son los sólidos sedimentables (SS), otros son los sólidos suspendidos totales (SST) y los sólidos suspendidos volátiles (SSV) (Avnimelech, 2009), así como el índice volumétrico de sólidos (SS x 1000 / SST) (Pasco, 2015). $100 \mathrm{ml} / \mathrm{L}$ de SS en cultivo de tilapia es a juicio de Avnimelech, 2009, un indicativo de buena calidad del biofloc. Para Rhamdia quelen los mejores resultados en cuanto a sobrevivencia y crecimiento de poslarvas y alevinos se consiguieron en concentración de SST de 200 mg/L (Poli et al., 2015), en tanto que para Ictalurus punctatus, los mejores resultados de su cultivo se encuentran en $790 \mathrm{mg} / \mathrm{L}$ (Green, 2015). Teniendo en cuenta la práctica de adición a los cultivos de hidratos de carbono y cal hidratada y que estos en conjunto con las raciones aumentan la concentración de SST, es necesario regularlos y controlarlos, con sedimentadores, por ejemplo como lo plantea Ray et al., (2010).

\section{Alcances actuales de la piscicultura con BFT}

El uso de biofloc para la piscicultura de tilapia ha conseguido incrementar las producciones de $2 \mathrm{~kg} / \mathrm{m}^{2}$ (cultivos semi-intensivos), a valores cercanos a $20 \mathrm{~kg} / \mathrm{m}^{2}$
(Hargreaves, 2013; Azim y Little, 2008; Avnimelech, 2007), hasta $30 \mathrm{~kg} / \mathrm{m}^{2}$ (Avnimelech, 2012).

En especies nativas, se reportan trabajos desarrollados en levante y engorde de cachama blanca con una biomasa final de $\approx 13 \mathrm{Kg} / \mathrm{m}^{3}$, iniciando con ejemplares de $48 \mathrm{~g}$ y finalizando con un peso promedio de 450 g en 192 días (Poleo et al., 2011). Abad et al., (2014), reportan $7,9 \mathrm{~kg} / \mathrm{m}^{3}$ al final del cultivo (210 días), con pesos finales promedio por ejemplar de $455 \mathrm{~g}$.

Los reportes de larvicultura y alevinaje en sistemas de biofloc en especies de peces son escasos, para tilapia nilotica (Oreochromis niloticus) Ekasari et al., (2015), obtuvieron sobrevivencias $>98 \%$, con larvas producidas y cultivadas en biofloc $(\mathrm{C} / \mathrm{N} 10)$, que con larvas en sistemas sin biofloc (sobrevivencias < $95 \%$ ); de igual manera las larvas cultivadas en biofloc fueron más resistentes y obtuvieron mejores sobrevivencias al ser confrontadas a pruebas de suspensión en medio con Streptococcus agalactiae $\left(10^{7} \mathrm{UFC} / \mathrm{ml}\right)$, por 6 horas y pruebas de estrés a altas salinidades $(35 \mathrm{~g} / \mathrm{L} \mathrm{NaCl})$, (sobrevivencia del $80 \%$ y $70 \%$ respectivamente).

En especies nativas suramericanas Poli et al., (2015), en pos-larvas de Rhamdia quelen, a densidad 25 poslarvas/L y diferentes concentraciones de sólidos totales en suspensión (SST), obteniendo los mejores resultados en cuanto a sobrevivencia y crecimiento a SST de $200 \mathrm{mg} / \mathrm{L}$.

Como se puede deducir de la literatura estudiada y referida en esta revisión, la BTF ha sido probada y desarrollada para tilapias, pero se requieren experimentos más apropiados a las condiciones del país y cálculos más detallados de los costos de producción, en especial los gastos por energía eléctrica, pero es sin ninguna duda una alternativa para los sistemas de producción intensiva de tilapia en Colombia. Aplicar la BTF la a otras especies requiere tenerse en cuanta las diferencia metabólicas de estas, así por ejemplo 50 $\mathrm{Kg}^{-1}$ de PV de cachama blanca con un peso promedio de $520 \mathrm{~g}$, alimentadas con un concentrado con $30 \%$ de PB, excretan 18,71 g de NAT * día ${ }^{-1}$ (David, 2009), mientras que $50 \mathrm{Kg}^{-1}$ de PV de tilapia bajo condiciones similares excretan 36 g de NAT * día ${ }^{-1}$ (De Schryver, 2008), el doble que cachama. Finalmente, se considera que la BFT puede ser una alternativa para la producción súper-intensiva de peces nativos. Sin embargo, se hacen necesarias investigaciones encaminadas a resolver las condiciones del biofloc propias para cada especie, biomasa de cultivo, estrategias de aireación para cada una de las diferentes etapas de desarrollo de las especies y de esta manera potenciar las posibilidades y optimizar los cultivos. 


\section{Referencias}

Abad D, Rincón D, Poleo G. Índices de rendimiento corporal en morocoto Piaractus brachypomus cultivado en sistemas Biofloc. Zootecnia Trop. 2014;32(2): 119-130.

Abreu PC, Ballester ELC, Odebrecht C, Wasielesky WJr, Cavalli RO, Granéli W, Anésio AM. Importance of biofilm as food source for shrimp (Farfantepenaeus paulensis) evaluated by stable isotopes (d13C and d15N). J Expl Mar Biol Ecol. 2007;347:88-96.

Atencio GV, Pertuz BV, Bru CS, Ayazo GJ. 2013. Curso teórico práctico tecnología de cultivo biofloc: fundamentos y manejo. Centro de Investigación Piscícola de la Universidad de Córdoba - CINPIC. Montería - Colombia.

AUNAP - Autoridad Nacional de Acuicultura y Pesca. 2013. Diagnóstico del estado de la acuicultura en Colombia. Bogotá, Colombia.

Avnimelech Y. Carbon nitrogen ratio as a control element in aquaculture systems. Aquaculture. 1999;176:227-235.

Avnimelech Y. Bio-filters: The need for an new comprehensive approach. Aquacult Eng. 2006;34:172-178.

Avnimelech Y. Feeding with microbial flocs by tilapia in minimal discharge bioflocs technology ponds. Aquaculture. 2007;264:140-147.

Avnimelech Y. 2009. Biofloc Technology - A practical Guide Book. The World Aquaculture Society. 272 pp.

Avnimelech Y. 2011. Tilapia Production Using Biofloc Technology Saving Water, Waste Recycling Improves Economics. Global aquaculture advocate May/June: 66-68. USA.

Avnimelech Y. 2012a. Nitrogen Isotope: Tool To Evaluate Protein Uptake In Biofloc Systems. Global Aquaculture Alliance. Marzo/Abril 2012. 74-75 pp.

Avnimelech Y. 2012b. Biofloc Technology - A Pratical Guide Book. The World Aquaculture Society, Baton Rouge, Louisiana, United States. 2. Ed.

Avinmelech Y, Verdegem MCJ, Kurup M, Keshavanath P. Sustainable land-based aquaculture: Rational utilization of water, land and feed resources. Med Aquacult J. 2008;1:45-55.

Azam F, Fenchel T, Field JG, Gray JS, Meyer-Reil LA, Thingstad F. The ecological role of water-column microbes in the sea. Mar Ecol Prog Ser. 1983;10:257-263.

Azim ME, Little DC. The bio $\square$ oc technology (BFT) in indoor tanks: Water quality, bio $\square$ oc composition, and growth and welfare of Nile tilapia (Oreochromis niloticus). Aquaculture. 2008;283:2935.

Azim ME, Little DC, Bron J. Microbial protein production in activated suspension tanks manipulating $\mathrm{C}: \mathrm{N}$ ratio in feed and implications for fish culture. Bioresour Technol. 2008;99(9):3590-3599.

Boyd C, Pond water aeration systems. Aquac Eng. 1998;18:9-40.

Boyd CE, Clay JW. 2002. Evaluation of Belize Aquaculture, Ltd: A Superintensive Shrimp Aquaculture System. Report prepared under the World Bank, NACA, WWF and FAO Consortium Program on Shrimp Farming and the Environment. 17pp.
Browdy C, Bratvold D, Stokes A, Mcintosh R. 2001. Perspectives on the application of closed shrimp culture systems. In: C.L. Browdy and D.E. Jory, (Eds.) The New Wave, Proceedings of the Special Session on Sustainable Shrimp Culture, Aquaculture The World Aquaculture Society, p. 20-34.

Burford MA, Thompson PJ, Mclntosh RP, Bauman RH, Pearson DC. Nutrient and microbial dynamics in high-intensity, zero-exchange shrimp ponds in Belize. Aquaculture. 2003;219:393-411.

Burford MA, Thompson PJ, Mclntosh RP, Bauman RH, Pearson DC. The contribution of flocculated material to shrimp (Litopenaeus vannamei) nutrition in a high-intensity, zero-exchange system. Aquaculture. 2004;232:525-537.

Chamberlain G, Avnimelech Y, Mclntosh R, Velasco M. Advantages of aerated microbial reuse systems with balanced C:N. III: practical applications. Global Aqua Advocate. 2001;4:50-54.

Collazos LLF y Arias CJA. Influencia de la temperatura en la sobrevivencia de larvas de Rhamdia sebae c.f. (Siluriformes heptapteridae). Orinoquia. 2007;11(1): 56-62.

Crab R, Avnimelech Y, Defoirdt T, Bossier P, Verstraete W. Nitrogen removal techniques in aquaculture for a sustainable production. Aquaculture 2007;270:1-14.

Crab R, Chielens B, Wille M, Bossier P, Verstraete W. The effect of different carbon sources on the nutritional value of bio $\square$ ocs, a feed for Macrobrachium rosenbergii postlarvae. Aquacult Res. 2010;41:559-567.

Crab R, Defoirdt T, Bossier P y Verstraete W. Bio $\square$ oc technology in aquaculture: Bene $\square$ cial effects and future challenges. Aquaculture. 2012;(356-357):351-356.

Craig LB, Andrew JR, John WL, Avnimelech Y. Bio $\square$ oc-based Aquaculture Systems. Aquaculture Production Systems, First Edition. Edited by James Tidwell. 2012;12:278-306.

Craig S, Helfrich LA. 2002. Understanding Fish Nutrition, Feeds and Feeding (Publication 420-256). Virginia Cooperative Extension, Yorktown (Virginia). 4 pp.

David RCA. 2009. Cuantificación de los niveles de excreción de nitrógeno amoniacal en función del nivel de proteína en la dieta y la masa corporal, en cachama blanca (Piaractus brachypomus) (Cuvier 1818) bajo condiciones de laboratorio. Tesis de maestría en Acuicultura. Universidad de los Llanos, Villavicencio Colombia.

De Schryver P, Crab R, Defoirdt T, Boon N, Verstraete W. The basics of bio- $\square$ ocs technology: The added value for aquaculture. Aquaculture. 2008;277:125-137.

Ebeling JM, Timmons MB, Bisogni JJ. Engineering analysis of the stoichiometry of photoautotrophic, autotrophic and heterotrophic removal of ammonia-nitrogen in aquaculture systems. Aquaculture. 2006;257:346-358.

Ekasari J, Deasy A, Waluyo SH, Bachtiar T, Surawidjaja EH, Bossier $\mathrm{P}$, De Schryver P. The size of bio $\square$ oc determines the nutritional composition and the nitrogen recovery by aquaculture animals. Aquaculture. 2014;(426-427):105-111.

Ekasari J, Rivandi DR, Firdausi AP, Surawidjaja EH, Zairin Jr M, Bossier, De Schryver P. Biofloc technology positively affects Nile 
tilapia (Oreochromis niloticus) larvae performance. Aquaculture. 2015;441:72-77.

Emerenciano M, Cuzon G, Goguenheim J, Gaxiola G, Aquacop. Floc contribution on spawning performance of blue shrimp Litopenaeus stylirostris. Aquac Res. 2012;44(1):75-85.

Emerenciano M, Gaxiola G y Cuzon G. 2013. Biofloc Technology (BFT): A Review for Aquaculture Application and Animal Food Industry. INTECH open science_open minds. Cap 12: 301327. http://dx.doi.org/10.5772/53902.

Emerson K, Russo RC, Lund RE, Thurston RV. Aqueous ammonia equilibrium calculations: effect of $\mathrm{pH}$ and temperature. J Fish Res Board Can. 1975;32:2379-2383.

FAO - Organización de las Naciones Unidas para la alimentación y la agricultura. 2012. El estado mundial de la pesca y la acuicultura. Roma.

FAO - Organización de las Naciones Unidas para la alimentación y la agricultura.2014. El estado mundial de la pesca y la acuicultura. Roma.

Gelineau A, Medale F. y Boujard T. Effect of feeding time on post prandial nitrogen excretion and energy expenditure in rainbow trout. J Fish Biol. 1998;52:655-664.

Green BW. Performance of a temperate-zone channel Cat fish biofloc technology production system during winter. Aquacultural Engineering. 2015;64:60-67.

Hargreaves JA. Nitrogen biogeochemistry of aquaculture ponds. Review. Aquaculture. 1998. 166 181-212.

Hargreaves JA. Photosynthetic suspended-growth systems in aquaculture. Aquacult Eng. 2006;34:344-363.

Hargreaves JA. Bioflóc Production Systems for Aquaculture. En: SRAC. Abril, 2013:4503:8-10.

Hari B, Kurup BM, Varghese JT, Schrama JW, Verdegem MCJ. Effects of carbohydrate addition on production in extensive shrimp culture systems. Aquaculture. 2004;241:179-194.

Hernández J, Vargas AF. A microplate technique to quantify nutrients ( $\mathrm{NO}_{2}=, \mathrm{NO}_{3}=, \mathrm{NH} 4+$ and $\left.\mathrm{PO} 43-\right)$ in seawater. Aquac Res. 2003;34:1201-1204.

Jorand F, Zartarian F, Thomas F, Block J, Bottero J, Villemin G, Urbain V, Manem J. Chemical and structural (2d) linkage between bacteria within activated-sludge flocs. Water Res. 1995;29(7):1639-1647.

Kubitza F. Criação de tilapias em sistema com bioflocos sem renovação de agua. Panorama da AQÜICULTURA. 2011;21(125):1423.

Kuhn D, Lawrence A. 2012. Biofloc Technology Options For Aquaculture In-Situ, Ex-Situ Systems Improve Water Quality, Provide Nutrition. Copyright (C) 2012, Global Aquaculture Alliance.

Kuhn D, Boardman G, Lawrence A, Marsh L, Flick G. Microbial floc meal as a replacement ingredient for fish meal and soybean protein in shrimp feed. Aquaculture. 2009;296:51-57.
Martínez CLR, Martínez PM, López EJA, Campaña TCA, Miranda BA, Ballester E, Porchas CMA, Martínez-Córdova L. 2010. Alimento Natural en Acuacultura: una revisión actualizada. En: Cruz-Suarez LE, Ricque-Marie D, Tapia-Salazar M, Nieto-López MG, Villarreal-Cavazos DA, Gamboa-Delgado J. (Eds). Avances en nutrición Acuícola X - Memorias del X Simposio Internacional de Alimento natural en acuacultura: Nutrición Acuícola, 8-10 de Noviembre, San Nicolás de los Garza, N. L., México. ISBN 978-607-433-546-0. Univ Autó Nvo León, Monterrey, México, pp. 668-699.

Mcintosh R, 2001. Changing Paradigms in Shrimp Farming. V: Establishment of heterotrofic bacterial commuinities. Global Aquaculture Alliance. v. February.

Monroy DMC, De Lara AR, Castro MJ, Castro MG y Emerenciano CM. Composición y abundancia de comunidades microbianas. Rev Biol Mar Oceanogr. 2013;48(3):511-520.

Moss S. 2002. Dietary importance of microbes and detritus in penaeid shrimp aquaculture, pp.1-18. In: Microbial Approaches to Aquatic Nutrition within Environmentally Sound Aquaculture Production Systems, CS Lee and P. O'Bryen (editors). The World Aquaculture Society, Baton Rouge, Louisisana, USA, 2002.

Okabe S, Watanabe Y. Structure and function of growth culture evaluation of Daphnia magna feed with Saccharomyces cereviseae enrichment with oat soy nitrifying biofilms as determined by in situ hybridization and the presence of microelectrodes. Water Sci Technol. 2000;42:21-32.

Pasco MJ. 2105. Aeraçãoem cultivos superintensivos de tilapias Oreochromis niloticus, em bioflocos e com troca mínima de água. Tese Doutorado em aquicultura. Universidade Federal De Santa Catarina, Centro De CiênciasAgrárias Departamento De Aquicultura, Florianapolis. Brasil.

Piedrahita RH. Reducing the potential environmental impact of tank aquaculture effluents through intensification and recirculation. Aquaculture. 2003;226:35-44.

Poleo G, Aranbarrio JV, Mendoza L, Romero O. Cultivo de cachama blanca en altas densidades y en dos sistemas cerrados. Pesq Agropec Bras. Brasília. 2011; 46(4):429-437.

Poli MA, Schveitzer R, Oliveira N. The use of biofloc technology in a South American catfish (Rhamdia quelen) hatchery: Effect of suspended solids in the performance of larvae. Aquacult Eng. 2015;66:17-21.

Ray AJ, Seaborn G, Leffler JW, Wilde SB, Lawson A, Browdy CL. Characterization of microbial communities in minimal-exchange, intensive aquaculture systems and the effects of suspended solids management. Aquaculture. 2010;310:130-138.

Ray JA, Lotz JM. Comparing a chemoautotrophic-based biofloc system and three heterotrophic-based systems receiving different carbohydrate sources. Aquacult Eng. 2014;63:54-61.

Sagratzki CBA, Pereira-Filho M, Bordinhon A, Fonseca FA, Ituassú D, Roubach R. y Ono EA. Tolerância de juvenis de pirarucuao aumento da concentração de amôniaem ambiente confinado. Pesq Agrop Bras. 2004;39:513-516.

Samocha TM, Patnaik S, Speed M, Ali AM, Burger JM, Almeida RV, Ayub Z, Harisanto M, Horowitz A, Brock DL. Use of molasses 
as carbon source in limited discharge nursery and grow-out systems for Litopenaeus vannamei. Aquacult Eng. 2007;36:184191

Sastre OF, Hernández G, Cruz CP. Influencia del peso corporal y de la temperatura del agua sobre el consumo de oxígeno de la Cachama Blanca (Piaractus brachypomus). Rev Col Cienc Pec. 2004; 17:11-16.

Schneider O, Sereti V, Eding EP y Verreth JAJ. Molasses as C source for heterotrophic bacteria production on solid fish waste. Aquaculture. 2006;261:1239-1248.

Schveitzer R, Arantes R, Costódio PFS, do Espírito Santo CM, Arana LV, Seiffert WQ, Andreatta ER, Effect of different biofloc levels on microbial activity, water quality and performance of Litopenaeus vannamei in a tank system operated with no water exchange. Aquacult Eng. 2013;56:59-70.

Timmons MB, Ebeling JM, Wheaton FW, Sommerfelt ST, Vinci BJ. 2002. Microbial biofloc and protein levels in green tiger shrimp. Recirculating aquaculture systems, 748 pp. Caruga Aqua Ventures, New York.

Timmons MB, Ebeling JM, Wheaton FW, Summerrfelt ST, Vinci BJ.2002a. Recirculating aquaculture systems. 2 ed. New York: Cayuga Aqua Venture, 769 pp

Timmons MB y Ebeling JM. 2010. Recirculating Aquaculture. NRAC Publication No. 401. Ithaca, NY, 948 pp.
Tzachi M, Samocha BA, Correia ES, Morris TC, Wilkenfeld JS. 2012. Growth performance of Litopenaeus vannamei in super-intensive mixotrophic raceway culture with zero discharge using Tareation ${ }^{\circledR}$ technology for aeration and extended $\mathrm{CO}_{2}$ degassing. TexasAgriLife Research Mariculture Lab. at Flour Bluff, Corpus Christi, Texas. 45p.

Valbuena M, Velasco SY, Cruz CP. Efecto del peso corporal sobre el consumo de oxígeno en yamú (Brycon amazonicus Spix \& Agassiz 1829): reporte preliminar. Rev Col Cienc Pec. 2006;19(2):175-179.

Valbuena RD, Cruz CP. Efecto del peso corporal y temperatura del agua sobre el consumo de oxígeno de tilapia roja (Oreochromis sp). Orinoquia. 2006;10(1):57-63.

Vinatea L. 2004. Principios químicos de qualidade da agua em aqüicultura. 2. ed: Editora da UFSC, Florianópolis 345 pp.

Wasielesky WJr, Atwood H, Stokes A, Browdy CL. Effect of natural production in a zero exchange suspended microbial floc based super-intensive culture system for white shrimp Litopenaeus vannamei. Aquaculture. 2006;258:396-403.

WHO - World Health Organization technical report. 2003. series № 916.150pp.

Wilén BM, Onuki M, Hermansson M, Lumley D, Mino T. Microbial community structure in activated sludge floc analysed by fluorescence in situ hybridization and its relation to floc stability. Water Research, 2008;42(8-9): 2300-2308. 